Research Paper

\title{
HER2 as a Prognostic Marker in Gastric Cancer - A Systematic Analysis of Data from the Literature
}

\author{
Jan Trøst Jørgensen ${ }^{1 凶}$, Maria Hersom²
}

1. Dx-Rx Institute, Baunevaenget 76, DK-3480 Fredensborg, Denmark;

2. Human Biology, Faculty of Health Sciences, University of Copenhagen, DK-2200 Copenhagen, Denmark.

$\checkmark$ Corresponding author: Dx-Rx Institute, Baunevaenget 76, DK-3480 Fredensborg, Denmark, Phone: +45 4074 7846 , Fax: +458870 8090, E-mail: jan.trost@dx-rx.dk.

() Ivyspring International Publisher. This is an open-access article distributed under the terms of the Creative Commons License (http://creativecommons.org/ licenses/by-nc-nd/3.0/). Reproduction is permitted for personal, noncommercial use, provided that the article is in whole, unmodified, and properly cited.

Received: 2012.01.14; Accepted: 2012.03.04; Published: 2012.03.12

\begin{abstract}
Through the recent conduct of the ToGA trial, HER2 has shown to be predictive for the treatment with trastuzumab in advanced gastric and gastro-oesophageal cancer. When it comes to the prognostic properties the situation is different. Despite the fact that it is more than 20 years ago since the first studies demonstrating an association between a positive HER2 status and poor prognosis were published the issue is still controversial. In this current systematic review a large number of studies on HER2 and gastric cancer have been reviewed. The studies included in this review should fulfill the following two criteria. First criterion: The number of patients in each study should be $\geq 100$, and the HER2 status should have been determined either by immunohistochemistry (IHC) or in situ hybridization (ISH). Second criterion: The selected articles should include an analysis of the association between the HER2 status and survival or relevant clinicopathological characteristics. Forty-two publications with a total of I2,749 patients fulfilled the two criteria and were reviewed in detail. The majority of the publications (7I\%) showed that a HER2-postive status measured either by IHC or ISH was associated with poor survival and/or clinicopathological characteristics, such as serosal invasion, lymph node metastases, disease stage, or distant metastases. Based on the current analysis a clear trend towards a potential role for HER2 as a negative prognostics factor in gastric cancer was shown, suggesting that HER2 overexpression and/or amplification is a molecular abnormality that might be linked to the development of gastric cancer.
\end{abstract}

Key words: HER2, gastric cancer, prognostic marker, IHC, FISH, CISH.

\section{Introduction}

HER2 has shown to possess predictive properties for the treatment with the monoclonal antibody trastuzumab (Herceptin, Roche/Genentech) in breast cancer, and the recent ToGA trial has shown similar characteristic in gastric cancer [1-3]. However, when it comes to the prognostic properties of the HER2 status there seems to be differences in how the marker is perceived in the two diseases. For breast cancer HER2 was found to be a negative prognostic factor very early on [4], but for gastric cancer there still seems to be no consensus, despite the fact that the first studies demonstrating an association between a positive HER2 status and poor prognosis appeared already 20 years ago $[5,6]$.

The gene for the HER2 protein (also known as ErbB-2, c-erbB2 or Her2/neu) is a proto-oncogene located on the chromosome 17. The HER2 gene encodes a $185-\mathrm{kDa}$ transmembrane tyrosine kinase receptor protein, which is a member of the HER-family (HER1-4) [1, 7]. HER2 amplification and overexpression have been found to promote tumorigenesis and to be involved in pathogenesis of several human 
cancers [1, 3]. Slamon et al. demonstrated that amplification of the HER2 gene in patients with breast cancer negatively impacted the recurrence of the disease and the survival [4]. For both gene amplification and overexpression of the protein similar results have subsequently been shown in a large number of studies. A recent meta-analysis, based on data from 39,730 patients, has further confirmed the prognostic properties of a positive HER2 status in women with breast cancer [1].

For gastric or gastro-oesophageal junction cancer the prognostic significance of the HER2 status seems less clear and it has recently been discussed in relation to the publication of the results from the ToGA trial [2]. In this study the patients with HER2 positive disease were randomized to receive chemotherapy (capecitabine (Xeloda, Roche) plus cispaltin or fluorouracil plus cisplatin) or chemotherapy plus trastuzumab. The primary analysis of the study showed a median overall survival (OS) of 13.8 month for the group of patients that received the combined treatment of chemotherapy and trastuzumab compared to 11.1 month for the group that was assigned to chemotherapy alone. In the discussion section of the paper recently published in the Lancet it was mentioned that the OS of 11.1 month in the group of patients receiving chemotherapy alone was longer than expected. As a possible explanation for this it was stated that HER2 overexpression might already be conferring a better prognosis across the groups of patients studied. However, it was also mentioned that HER2 overexpression leading to a better prognosis is in contrast to recent studies that have showed an association between HER2-positive tumors and poor outcome and aggressive disease. The authors further concluded that more studies are needed to address the issue of whether HER2 has an effect on prognosis in gastric cancer, and whether it confers a good or poor prognosis [2].

For more than 20 years a relatively large number of studies on HER2 in gastric cancer have been published, and many of these have looked at the association between a HER2-postive status and survival and/or important clinicopathological characteristics. In this current systematic review we have utilized the already existing literature to address the issue on the prognostic properties of a HER2-postive status in gastric cancer.

\section{Methods}

A PubMed search was undertaken to identify articles in English, from 1986 to August 2011, using the key word "gastric cancer" combined with "HER2", "ErbB-2", "c-erbB2", and "Her2/neu". Ad- ditional articles were found by a manual search for references from the already identified articles. The articles were included in the analysis, if they fulfilled the following two criteria. Criterion 1: The number of patients studied should be $\geq 100$ and the HER2 status should have been determined by immunohistochemistry (IHC) or in situ hybridization (ISH) such as fluorescence in situ hybridization (FISH) or chromogenic in situ hybridization (CISH). Criterion 2: The selected articles should include an analysis of the association between the HER2 status and survival, e.g. OS and/or disease-free survival (DFS) and/or relevant clinicopathological characteristics such as serosal invasion, vascular invasion, lymph node involvement, distant metastases, disease stage, etc. Examples of the staining methods related to Criterion 1 are shown in Figure 1.

The identified articles were scored according to the strength of the association between the positive HER2 status and the prognostic information reported, using a three point categorical scale. If a statistic significant association between the HER2-positive status and survival was reported, the studies were marked with two pluses $(++)$ indicating the strongest association. For the studies where a statistic significant association between the HER2-postive status and relevant clinicopathological characteristics was found, the study was marked with one plus (+) indicating a somewhat weaker association. The studies were marked with a minus (-) where no association with a HER2-positive status was found. Separate tables were made for IHC or ISH and a weighted mean of the HER2-positive status was calculated together with the $95 \%$ confidence interval (CI) for each of the two assay type.

\section{Results}

For the studies conducted with IHC, 48 articles comprising 17,338 patients that fulfilled selection Criterion 1 were identified, and among these 38 articles comprising 11,860 patients also fulfilled Criterion 2. The weighted mean HER2-positive status for the articles that fulfilled both selection criteria was $17.9 \%$ ( $95 \%$ CI; 14.8 to 20.9 ). The corresponding range for the HER2-positive status was from $4.4 \%$ to $53.4 \%$. Two out of the 38 articles comprised patients both with gastroesophageal junction and gastric cancer [8, 9], and for the patients in the remaining articles the cancer was localized to the stomach. The individual studies and the association between HER2 overexpression and survival and/or relevant clinicopathological characteristics are listed in Table 1 scored according to the 3 point categorical scale as described above. The article by $\mathrm{H}$ Grabsch et al. [10] included 
two distinct populations, one from Germany and one from the UK, and these two populations have been treated separately and appear as two studies in Table 1.

In 15 of the 39 studies (39\%) an association was found between a positive HER2 status and poor survival $(++)$, and a similar association with clinicopathological characteristics $(+)$ was found in an additional 13 studies (33\%). In the remaining 11 studies (28\%) no association could be detected between a positive HER2 status and neither poor survival nor clinicopathological characteristics (-). In total for the IHC articles, 28 out of the 39 studies (72\%) showed an association between a positive HER2 status and poor survival or clinicopathological characteristics. In the individual studies the analysis of the association between HER2 status and survival or clinicopathological characteristics were performed using both univariate and multivariate statistics. Twenty of the 39 studies used both univariate and multivariate statistics and for the remaining 19 studies only univariate statistics was used. For the studies where an association between HER2 status and survival was demonstrated $(++), 11$ of the 15 studies in this category used multivariate statistics.

For the studies categorized as $(+)$ or $(++)$ the clinicopathological characteristics that was most often found to be associated with a positive HER2 status was lymph node status, serosal invasion or the depth of invasion, disease stage, and distant metastases. Further, for all the IHC studies $(-/+/++)$ an association between overexpression of HER2 and the intestinal tumor type was found in more than half of these studies. When the studies where the positive HER2 status showed no prognostic value (-) were compared with the studies where the positive status correlated with poor survival or clinicopathological characteristics $(++/+)$ there was a trend towards a lower HER2 positivity rate for the "negative" studies. The weighted mean for the "negative" studies was 15.4\% (95\% CI; 9.4 to 19.2 ) compared to $18.2 \%$ (95\% CI; 14.5 to 22.0) for the positive studies, however, as shown the difference was not statistically significant.

For the studies that included ISH testing, 12 articles comprising 5,490 patients that fulfilled selection Criterion 1 were indentified, and among these 8 articles comprising 1,597 patients also fulfilled Criterion 2. The weighted mean for the HER2-positive status (amplification) for the articles that fulfilled both selection criteria was $12.2 \%$ (95\% CI; 9.5 to 14.8). The corresponding range for the HER2-positive status was from $8.7 \%$ to $18.1 \%$. Two out of the 8 articles comprised patients with both gastroesophageal junction and gastric cancer $[9,13]$, and for the patients in the remaining articles the cancer was localized to the stomach. The individual studies and the association between HER2 amplification and survival and/or relevant clinicopathological characteristics are listed in Table 2 scored according with the 3 point categorical scale. In 5 of the listed studies $[9,11,12,18,24]$ the HER2 status was determined by both IHC and ISH, and these articles are thus listed in Table 1 as well as in Table 2.

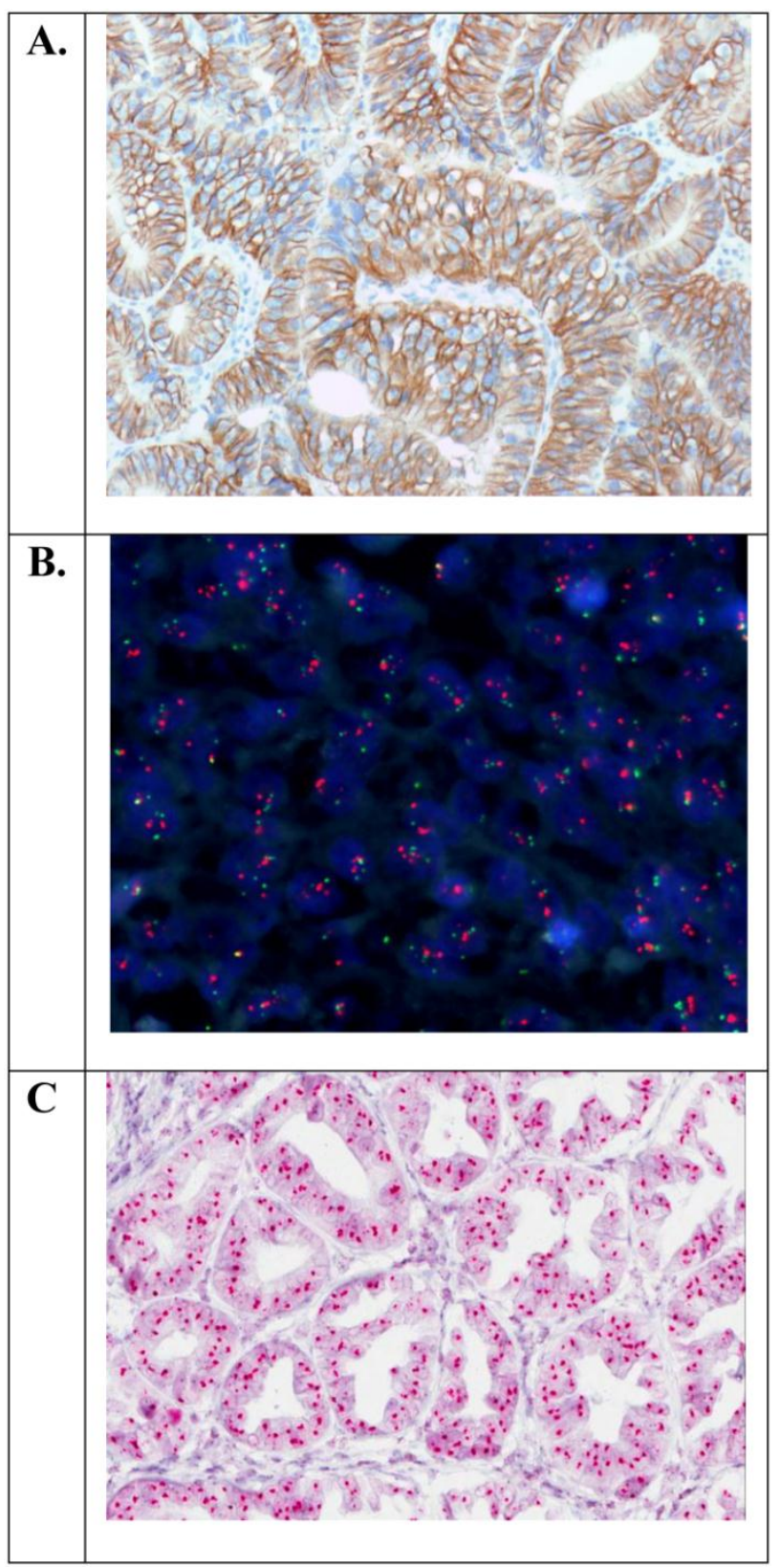

Figure I. Examples of positive HER2 status in gastric adenocarcinoma of the stomach with overexpression of the HER2 protein $(\mathrm{IHC} 3+)$ or amplification of the HER2 gene (HER2/CEN-I7 $\geq 2.0)$. A. Immunohistochemistry (HercepTest ${ }^{\mathrm{TM}}$, Dako $(20 \times)$ ). B. Fluorescence in situ hybridization (HER2 FISH pharmDx ${ }^{\text {TM }}$ Kit, Dako $(40 \times))$. C. Chromogenic in situ hybridization (HER2 CISH pharmDx ${ }^{\mathrm{TM}}$ Kit, Dako (40x)). 
Table I. The individual IHC studies fulfilling both Criteria I and 2.

\begin{tabular}{|c|c|c|c|c|c|}
\hline No. & Study & Country & $\mathrm{N}$ & HER2+ \% & Prognostic Info. \\
\hline 1 & Y Yonemura et al. (1991) [5] & Japan & 260 & 11.9 & ++ \\
\hline 2 & J K Roh et al (1992) [6] & Korea & 109 & 31.0 & ++ \\
\hline 3 & M Tateishi et al. (1992) [31] & Japan & 179 & 12.0 & - \\
\hline 4 & Y Yonemura et al. (1992) [32] & Japan & 220 & 10.0 & + \\
\hline 5 & T Mizutani et al. (1993) [33] & Japan & 226 & 14.0 & ++ \\
\hline 6 & T Ohguri et al. (1993) [34] & Japan & 186 & 30.6 & - \\
\hline 7 & S Uchino et al. (1993) [35] & Japan & 108 & 11.0 & ++ \\
\hline 8 & J-P Kim et al. (1994) [36] & Korea & 152 & 9.2 & - \\
\hline 9 & K Motojima et al. (1994) [37] & Japan & 120 & 28.0 & ++ \\
\hline 10 & S Chariyalertsak et al. (1994) [38] & Thailand & 309 & 11.7 & + \\
\hline 11 & A Webb et al. (1996) [39] & UK & 160 & 8.0 & + \\
\hline 12 & H R Lee et al. (1996) [40] & Korea & 225 & 27.4 & + \\
\hline 13 & C-T Shun et al. (1997) [16] & Taiwan & 112 & 30.3 & ++ \\
\hline 14 & M-S Wu et al. (1997) [17] & Taiwan & 163 & 26.4 & + \\
\hline 15 & T Ishikawa et al. (1997) [18] & Japan & 375 & 10.4 & + \\
\hline 16 & Y Yonemura et al. (1998) [19] & Japan & 299 & 13.7 & ++ \\
\hline 17 & M Nakajima et al. (1999) [20] & Japan & 128 & 16.4 & ++ \\
\hline 18 & J Sanz-Ortega et al. (2000) [41] & Spain & 143 & 26.6 & + \\
\hline 19 & A Ougolkov et al. (2000) [42] & Japan & 116 & 12.1 & - \\
\hline 20 & H Allgayer et al. (2000) [43] & Germany & 189 & 53.4 & ++ \\
\hline 21 & A Ghaderi et al. (2002) [44] & Iran & 146 & 16.4 & + \\
\hline 22 & T Takehana et al. (2002) [45] & Japan & 352 & 8.2 & + \\
\hline 23 & J Pinto-de-Sousa et al (2002) [46] & Portugal & 157 & 15.3 & ++ \\
\hline 24 & Y-L Wang et al. (2002) [47] & Taiwan & 100 & 32.0 & + \\
\hline 25 & KE Lee et al. (2003) [21] & Korea & 841 & 16.9 & - \\
\hline 26 & HS Song et al. (2003) [48] & Korea & 739 & 26.2 & - \\
\hline 27 & Z Tang et al. (2004) [49] & China & 200 & 5.5 & - \\
\hline 28 & DI Park et al. (2006) [22] & Korea & 182 & 15.9 & ++ \\
\hline 29 & AH Marx et al.(2009) [11] & Germany & 166 & 19.0 & - \\
\hline 30 & XL Zhang et al. (2009) [23] & China & 102 & 18.6 & ++ \\
\hline 31 & GZ Yu et al. (2009) [50] & China & 1143 & 28.0 & + \\
\hline 32 & H Grabsch et al. $(2010)^{*}[10]$ & Germany & 418 & 8.6 & - \\
\hline 33 & H Grabsch et al. $(2010)^{*}[10]$ & UK & 506 & 4.4 & - \\
\hline 34 & B Yan et al. (2010) [24] & Singapore & 128 & 9.4 & ++ \\
\hline 35 & S-Y Yan et al. (2011) [12] & China & 145 & 15.2 & + \\
\hline 36 & KC Kim et al. (2011) [25] & Korea & 2009 & 13.8 & ++ \\
\hline 37 & S-A Im et al (2011) [8] & Korea & 142 & 15.7 & + \\
\hline 38 & PL Kunz et al. (2011) [9] & USA & 169 & 13.0 & - \\
\hline 39 & Y-Y Wang et al. (2011) [26] & China & 436 & 20.6 & ++ \\
\hline
\end{tabular}

*This article comprises both a German and an English study population.

Symbols:

++ HER2 overexpression is associated with poor survival (e.g. OS, DFS, PFS, etc.)

+: HER2 overexpression is associated with clinicopathological characteristics (e.g. lymph node status, serosal invasion, disease stage, etc.)

-: $\quad$ No association found between HER2 overexpression and clinicopathological characteristics or poor survival. 
Table 2. The individual ISH studies fulfilling both Criteria I and 2.

\begin{tabular}{lllllll}
\hline No. & Study & Country & Assay & N & HER2 + \% & Prognostic Info. \\
\hline 1 & T Ishikawa et al. (1997) [18] & Japan & FISH & 105 & 18.1 & + \\
2 & M Tanner et al. (2005) [13] & Finland & CISH & 231 & 17.3 & ++ \\
3 & AH Marx et al.(2009) [11] & Germany & FISH & 166 & 16.0 & - \\
4 & JD Barros-Silva et al. (2009) [27] & Portugal & FISH & 463 & 9.3 & ++ \\
5 & B Yan et al. (2010) [24] & Singapore & FISH/CISH & 128 & 11.7 & ++ \\
6 & C B Moelans et al. (2011) [28] & Netherlands/Poland/ & CISH & 195 & 8.7 & - \\
7 & S-Y Yan et al. (2011) [12] & Finland/USA & FISH & 145 & 12.4 & + \\
8 & PL Kunz et al. (2011) [9] & China & FISH & 164 & 9.5 & - \\
\hline
\end{tabular}

Symbols:

++: HER2 amplification is associated with poor survival (e.g. OS , DFS, PFS, etc.)

+: $\quad$ HER2 amplification is associated with clinicopathological characteristics (e.g. lymph node status, serosal invasion, disease stage, etc.)

-: $\quad$ No association found between HER2 amplification and clinicopathological characteristics or poor survival.

In 3 of the 8 studies $(37.5 \%)$ an association was found between a positive HER2 status and poor survival (++) and in further 2 studies $(25 \%)$ a similar association was found with regards to clinicopathological characteristics $(+)$. In the remaining 3 studies $(37.5 \%)$ no association could be detected between a positive HER2 status and neither poor survival nor clinicopathological characteristics (-). In total for the ISH articles, 5 out of the 8 studies $(62.5 \%)$ showed an association between a positive HER2 status and poor survival or clinicopathological characteristics. For the individual studies the analysis of the association between HER2 status and survival or clinicopathological characteristics were performed using univariate statistical methods only.

For the studies categorized as $(+)$ or $(++)$ the clinicopathological characteristics that was most often found to be associated with a positive HER2 status was the same as mentioned for the IHC studies. Moreover, with respect to HER2 gene amplification an association with the intestinal tumor type was found in more than half of the studies. When the studies where the positive HER2 status showed no prognostic value $(-)$ was compared with the studies where the positive status correlated with poor survival or clinicopathological characteristics $(++/+)$ the HER2 positivity rate seemed to be similar. The weighted mean for the "negative" studies was 13.3\% (95\% CI; 6.7 to $15.8)$, and the corresponding value for the "positive" studies was $12.6 \%$ (95\% CI; 9.3 to 15.9 ).

If the IHC studies in Table 1 are pooled with the ISH studies in Table 2 the total number increases to 42 , comprising altogether 12,479 patients. The 5 studies $[9,11,12,18,24]$, where the HER2 status was determined by both IHC and ISH are only counted once. In 17 of these 42 studies $(\sim 40 \%)$ an association be- tween a positive HER2 status and poor survival $(++)$ was found, and an additional 13 studies $(\sim 31 \%)$ similarly showed a relationship with clinicopathological characteristics $(+)$. In the last 12 studies $(\sim 29 \%)$ no association between a positive HER2 status and poor survival or clinicopathological characteristics could be detected (-). Overall, this means that in 30 studies $(71 \%)$ an association between a positive HER2 status and poor survival and/or relevant clinicopathological characteristics could be found. Figure 2 illustrates the number of IHC and ISH studies and patients in each of the three scoring categories.

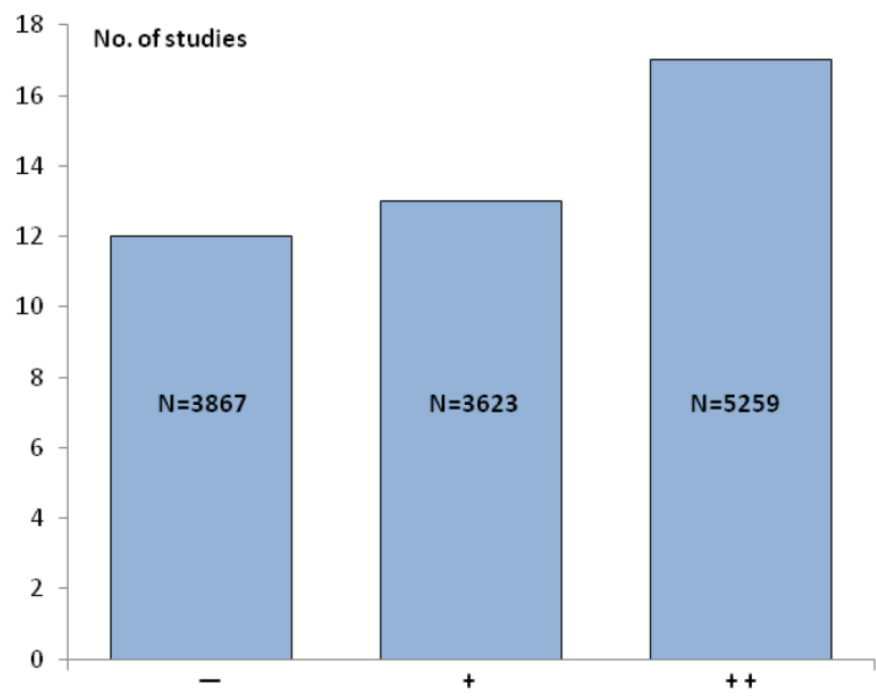

Figure 2. The number of IHC and ISH studies and patients $(\mathrm{N})$ in each of the three scoring categories. Symbols: Two pluses (++) indicate the strongest association with the HER2+ status, one plus $(+)$ indicates a somewhat weaker association with the HER2+ status, and minus ( -$)$ indicates that no associations with the HER2+ was found. 


\section{Discussion}

This systematic review has been based on 42 publications covering the period from 1991 to August 2011 and comprises a total of 12,749 patients with gastric cancer. The majority of the articles $(71 \%)$ reviewed showed that a HER2-postive status measured either by IHC or ISH provides additional prognostic information either related to survival $(40 \%)$ or to relevant clinicopathological characteristics (31\%), which included already established prognostic factors such as serosal invasion and lymph node metastases [14]. A clear trend towards a potential role for HER2 as a negative prognostic factor in gastric cancer was shown, although the data is not as consistent compared to what has been reported for breast cancer [1]. An issue that weakens the conclusion in this systematic review is the fact that multivariate statistics was only used in approximately half of the reported studies. However, it has recently been suggested that HER2 overexpression and/or amplification may be one of the molecular abnormalities linked to the development of gastric cancer, especially in the intestinal phenotype [15], and this support the data presented in this review. For the studies in the current review that have looked at the Lauren classification in relation to HER2, a higher level of overexpression or amplification was found in the intestinal phenotype compared to the diffuse or mixed types [8-12, 16-21, $23,25,27,28,41,44,46-48]$. A few of the IHC studies also seems to show that the expression of HER2 increased with disease progression [5, 33, 34, 38].

Based on the results of the current review it seems unlikely that HER2 overexpression or HER2 gene amplification could act as a positive prognostic factor in gastric cancer, as has been hypothesized in the publication of the ToGA trial in the Lancet [2]. None of the articles that fulfilled Criteria 1 and 2 in this analysis support this hypothesis. Possible confounding factors, such as a wider use of second line treatments and a possible better prognosis related to the intestinal phenotype, should be taken into consideration when interpreting the data from the ToGA trial [15]. With regards to the latter it is worth mentioning that approximately $75 \%$ of the patients included in the ToGA trial had tumors of the intestinal type [2], which seems to be high compared to most of the studies reported in this review. In support of the hypothesis put forward in the Lancet article one study has been identified, which, in fact, showed that overexpression of the HER2 resulted in a better prognosis compared to those who did not overexpress the protein. However, this study was not included in this review due to the number of patients being $<100$
(Criterion 1). Here, samples from 93 patients with advanced gastric carcinoma were investigated using IHC. Overexpression of HER2 was found in 10 patients $(11 \%)$ and a multifactorial analysis showed a significantly better prognosis for those patients in relation to survival [29].

A relatively large variation was found in the HER2 positivity rate between the individual studies included in this review as appears from Table 1 and Table 2. Here, the largest variation was seen in the IHC studies, where the range in relation to the percentage of patients with HER2 positive tumors was found to be between $4.4 \%$ and $53.4 \%$. The explanation for this rather large variation is likely to be multifactorial such as the difference in the populations studied, but the most important aspects are probably the use of non-standardized assays using different antibodies and the application of different scoring criteria for the stained slides. In the majority of the studies conducted within the last couple of years the scoring criteria developed by Hofmann et al. for the ToGA trial [30] or related were used, which seems to have resulted in less variation (range: $9.4 \%$ - 15.7) $[8,9,12$, $24,25]$. Also for the ISH studies some variation in relation to the percentage of patients with amplification of the HER2 gene (range: $8.7 \%-18.10 \%$ ) was found, although the magnitude is much smaller. This is likely explained by the fact that the FISH and CISH assays are quantitative methods compared to IHC, which use a semi-quantitative scoring system.

Despite the fact that the number of studies conducted with IHC is much higher than for ISH there seems to be no major differences in the results obtained. For the IHC articles, $72 \%$ of the studies (28 out of the 39) showed an association between HER2+ status and poor survival and/or clinicopathological characteristics $(++/+)$. The similar figures for the ISH studies were $63 \%$ ( 5 out of the 8 ). The trend towards a lower HER2 positivity rate for the "negative" studies that was observed for IHC may be explained by the large variation seen with this type of assay compared to ISH as described above.

\section{Conclusion}

The analysis of 42 publications comprising a total of 12,749 patients with gastric cancer showed that a positive HER2 status, measured either by IHC or ISH, adds information to the prognosis of the disease. Based on the current analysis a clear trend towards a potential role for HER2 as a negative prognostic factor in gastric cancer was shown, which suggests that HER2 overexpression and/or amplification might be a molecular abnormality linked to the development of gastric cancer. 


\section{Acknowledgments}

We would like to thank Dako Denmark A/S for their permission to use the microscopic gastric adenocarcinoma images.

\section{Competing Interests}

Jan Trøst Jørgensen has worked as a consultant for Dako Denmark A/S, Glostrup, Denmark and has given lectures at meetings sponsored by Roche and AstraZeneca. Maria Hersom has nothing to disclose.

\section{References}

1. Ross JS, Slodkowska EA, Symmans WF, et al. The HER-2 receptor and breast cancer: ten years of targeted anti-HER-2 therapy and personalized medicine. Oncologist 2009; 14: 320-368.

2. Bang YJ, Van Cutsem E, Feyereislova A, et al. Trastuzumab in combination with chemotherapy versus chemotherapy alone for treatment of HER2-positive advanced gastric or gastro-oesophageal junction cancer (ToGA): a phase 3, open-label, randomised controlled trial. Lancet 2010; 376: 687-697.

3. Jørgensen JT. Targeted HER2 treatment in advanced gastric cancer. Oncology 2010; 78: 26-33.

4. Slamon DJ, Clark GM, Wong SG, et al. Human breast cancer: correlation of relapse and survival with amplification of the HER-2/neu oncogene. Science 1987; 235: 177-182.

5. Yonemura Y, Ninomiya I, Yamaguchi A, et al. Evaluation of immunoreactivity for erbB-2 protein as a marker of poor short term prognosis in gastric cancer. Cancer Res 1991; 51: 1034-1038.

6. Roh JK, Paik S, Chung HC, et al. Overexpression of erbB-2 protein in gastric adenocarcinoma--a potential role in therapeutic response to adjuvant 5-FU-doxorubicin regimen. Jpn J Cancer Chemother 1992; 19: 1207-1219.

7. Coussens L, Yang-Feng TL, Liao YC, et al. Tyrosine kinase receptor with extensive homology to EGF receptor shares chromosomal location with neu oncogene. Science 1985; 230: 1132-1139.

8. Im SA, Kim JW, Kim JS, et al. Clinicopathologic characteristics of patients with stage III/IV $(\mathrm{M}(0))$ advanced gastric cancer, according to HER2 status assessed by immunohistochemistry and fluorescence in situ hybridization. Diagn Mol Pathol 2011; 20: 94-100.

9. Kunz PL, Mojtahed A, Fisher GA, et al. HER2 Expression in Gastric and Gastroesophageal Junction Adenocarcinoma in a US Population: Clinicopathologic Analysis With Proposed Approach to HER2 Assessment. Appl Immunohistochem Mol Morphol 2012 Jan;20(1):13-24.

10. Grabsch H, Sivakumar S, Gray S, et al. HER2 expression in gastric cancer: Rare, heterogeneous and of no prognostic value conclusions from 924 cases of two independent series. Cell Oncol 2010; 32: 57-65.

11. Marx AH, Tharun L, Muth J, et al. HER-2 amplification is highly homogenous in gastric cancer. Hum Pathol 2009; 40: 769-777.

12. Yan SY, Hu Y, Fan JG, et al. Clinicopathologic significance of HER-2/neu protein expression and gene amplification in gastric carcinoma. World J Gastroenterol 2011; 17: 1501-1506.

13. Tanner M, Hollmen M, Junttila TT, et al. Amplification of HER-2 in gastric carcinoma: association with Topoisomerase Ilalpha gene amplification, intestinal type, poor prognosis and sensitivity to trastuzumab. Ann Oncol 2005; 16: 273-278.
14. Shiraishi N, Sato K, Yasuda K, et al. Multivariate prognostic study on large gastric cancer. J Surg Oncol 2007; 96: 14-18.

15. Fornaro L, Lucchesi M, Caparello C, et al. Anti-HER agents in gastric cancer: from bench to bedside. Nat Rev Gastroenterol Hepatol 2011; 8: 369-383.

16. Shun CT, Wu MS, Lin JT, et al. Relationship of p53 and c-erbB-2 expression to histopathological features, Helicobacter pylori infection and prognosis in gastric cancer. Hepatogastroenterology 1997; 44: 604-609.

17. Wu MS, Shun CT, Wang HP, et al. Genetic alterations in gastric cancer: relation to histological subtypes, tumor stage, and Helicobacter pylori infection. Gastroenterology 1997; 112: 1457-1465.

18. Ishikawa $\mathrm{T}$, Kobayashi M, Mai M, et al. Amplification of the c-erbB-2 (HER-2/neu) gene in gastric cancer cells. Detection by fluorescence in situ hybridization. Am J Pathol 1997; 151: 761-768.

19. Yonemura $Y$, Ninomiya I, Tsugawa $K$, et al. Prognostic significance of c-erbB-2 gene expression in the poorly differentiated type of adenocarcinoma of the stomach. Cancer Detect Prev 1998; 22: 139-146.

20. Nakajima M, Sawada H, Yamada $Y$, et al. The prognostic significance of amplification and overexpression of c-met and c-erb B-2 in human gastric carcinomas. Cancer 1999; 85: 1894-1902.

21. Lee KE, Lee HJ, Kim YH, et al. Prognostic significance of p53, nm23, PCNA and c-erbB-2 in gastric cancer. Jpn J Clin Oncol 2003; 33: 173-179.

22. Park DI, Yun JW, Park JH, et al. HER-2/neu amplification is an independent prognostic factor in gastric cancer. Dig Dis Sci 2006; 51: 1371-1379.

23. Zhang XL, Yang YS, Xu DP, et al. Comparative study on overexpression of HER2/neu and HER3 in gastric cancer. World J Surg 2009; 33: 2112-2118.

24. Yan B, Yau EX, Bte Omar SS, et al. A study of HER2 gene amplification and protein expression in gastric cancer. J Clin Pathol 2010; 63: 839-842.

25. Kim KC, Koh YW, Chang HM, et al. Evaluation of HER2 Protein Expression in Gastric Carcinomas: Comparative Analysis of 1414 Cases of Whole-Tissue Sections and 595 Cases of Tissue Microarrays. Ann Surg Oncol 2011 Oct;18(10):2833-40.

26. Wang YY, Ye ZY, Li L, et al. ADAM 10 is associated with gastric cancer progression and prognosis of patients. J Surg Oncol 2011; 103: 116-123.

27. Barros-Silva JD, Leitao D, Afonso L, et al. Association of ERBB2 gene status with histopathological parameters and disease-specific survival in gastric carcinoma patients. Br J Cancer 2009; 100: 487-493.

28. Moelans CB, Milne AN, Morsink FH, et al. Low frequency of HER2 amplification and overexpression in early onset gastric cancer. Cell Oncol 2011; 34: 89-95.

29. Jain S, Filipe MI, Gullick WJ, et al. c-erbB-2 proto-oncogene expression and its relationship to survival in gastric carcinoma: an immunohistochemical study on archival material. Int J Cancer 1991; 48: 668-671.

30. Hofmann M, Stoss O, Shi D, et al. Assessment of a HER2 scoring system for gastric cancer: results from a validation study. Histopathology 2008; 52: 797-805.

31. Tateishi M, Toda T, Minamisono Y, Nagasaki S. Clinicopathological significance of c-erbB-2 protein expression in human gastric carcinoma. J Surg Oncol 1992; 49: 209-212.

32. Yonemura Y, Ninomiya I, Ohoyama S, et al. Correlation of c-erbB-2 protein expression and lymph node status in early gastric cancer. Oncology 1992; 49: 363-367.

33. Mizutani T, Onda M, Tokunaga A, et al. Relationship of C-erbB-2 protein expression and gene amplification to invasion 
and metastasis in human gastric cancer. Cancer 1993; 72: 2083-2088.

34. Ohguri T, Sato Y, Koizumi W, et al. An immunohistochemical study of c-erbB-2 protein in gastric carcinomas and lymph-node metastases: is the c-erbB-2 protein really a prognostic indicator? Int J Cancer 1993; 53: 75-79.

35. Uchino S, Tsuda H, Maruyama $K$, et al. Overexpression of c-erbB-2 protein in gastric cancer. Its correlation with long-term survival of patients. Cancer 1993; 72: 3179-3184.

36. Kim JP, Oh ST, Hwang TS, Chi JG. The prognostic significance of c-erbB-2 and p53 protein expressions in gastric carcinoma--a multivariate analysis of prognostic factors. J Korean Med Sci 1994; 9: 248-253.

37. Motojima K, Furui J, Kohara N, et al. erbB-2 expression in well-differentiated adenocarcinoma of the stomach predicts shorter survival after curative resection. Surgery 1994; 115: 349-354.

38. Chariyalertsak S, Sugano K, Ohkura H, Mori Y. Comparison of c-erbB-2 oncoprotein expression in tissue and serum of patients with stomach cancer. Tumour Biol 1994; 15: 294-303.

39. Webb A, Scott-Mackie P, Cunningham D, et al. The prognostic value of serum and immunohistochemical tumour markers in advanced gastric cancer. Eur J Cancer 1996; 32A: 63-68.

40. Lee HR, Kim JH, Uhm HD, et al. Overexpression of c-ErbB-2 protein in gastric cancer by immunohistochemical stain. Oncology 1996; 53: 192-197.

41. Sanz-Ortega J, Steinberg SM, Moro E, et al. Comparative study of tumor angiogenesis and immunohistochemistry for p53, c-ErbB2, c-myc and EGFr as prognostic factors in gastric cancer. Histol Histopathol 2000; 15: 455-462.

42. Ougolkov A, Mai M, Takahashi Y, et al. Altered expression of beta-catenin and c-erbB-2 in early gastric cancer. J Exp Clin Cancer Res 2000; 19: 349-355.

43. Allgayer H, Babic R, Gruetzner KU, et al. c-erbB-2 is of independent prognostic relevance in gastric cancer and is associated with the expression of tumor-associated protease systems. J Clin Oncol 2000; 18: 2201-2209.

44. Ghaderi A, Vasei M, Maleck-Hosseini SA, et al. The expression of c-erbB-1 and c-erbB-2 in Iranian patients with gastric carcinoma. Pathol Oncol Res 2002; 8: 252-256.

45. Takehana T, Kunitomo K, Kono K, et al. Status of c-erbB-2 in gastric adenocarcinoma: a comparative study of immunohistochemistry, fluorescence in situ hybridization and enzyme-linked immuno-sorbent assay. Int J Cancer 2002; 98: 833-837.

46. Pinto-de-Sousa J, David L, Almeida R, et al. c-erb B-2 expression is associated with tumor location and venous invasion and influences survival of patients with gastric carcinoma. Int J Surg Pathol 2002; 10: 247-256.

47. Wang YL, Sheu BS, Yang HB, et al. Overexpression of c-erb-B2 proteins in tumor and non-tumor parts of gastric adenocarcinoma--emphasis on its relation to $\mathrm{H}$. pylori infection and clinicohistological characteristics. Hepatogastroenterology 2002; 49: 1172-1176.

48. Song HS, Do YR, Kim IH, et al. Prognostic significance of immunohistochemical expression of EGFR and C-erbB-2 oncoprotein in curatively resected gastric cancer. Cancer Res Treat 2004; 36: 240-245.

49. Tang Z, Zhao M, Ji J, et al. Overexpression of gastrin and c-met protein involved in human gastric carcinomas and intestinal metaplasia. Oncol Rep 2004; 11: 333-339.

50. Yu GZ, Chen Y, Wang JJ. Overexpression of Grb2/HER2 signaling in Chinese gastric cancer: their relationship with clinicopathological parameters and prognostic significance. J Cancer Res Clin Oncol 2009; 135: 1331-1339. 